\title{
Photoelectric Charging of Dust Particles
}

\author{
A. Sickafoose, J. Colwell, M. Horányi, S. Robertson \\ Laboratory for Atmospheric and Space Physics, University of Colorado, Boulder, CO 80309-0392 \\ B. Walch
}

Department of Physics University of Northern Colorado, Greeley, CO 80639

\begin{abstract}
Laboratory experiments have been performed on the photoelectric charging of dust particles which are either isolated or adjacent to a surface that is also a photoemitter. We find that zinc dust charges to a positive potential of a few volts when isolated in vacuum and that it charges to a negative potential of a few volts when passed by a photoemitting surface. The illumination is an arc lamp emitting wavelengths longer than $200 \mathrm{~nm}$ and the emitting surface is a zirconium foil.
\end{abstract}

\section{INTRODUCTION}

Natural and man-made objects in space charge to a floating potential determined by the local plasma environment [1]. The dominant charging currents are the flux of electrons and ions from ambient plasma, electrons created by secondary emission and photoelectrons. An object floats at the potential at which the sum of the currents is zero. The charging within planetary magnetospheres is usually determined by the flux of magnetically trapped charged particles and secondaries. In interplanetary space and at geosynchronous orbit, on the other hand, the charging is usually dominated by photoelectric emission. In this case there is a positive floating potential at which nearly all of the photoelectrons are returned to the surface. This situation is altered only slightly by the small flux of solar wind particles. For objects of centimeter scale and larger, the local plasma environment is dominated by photoelectrons. Dust on larger objects such as the Moon [2] or asteroids $[3,4]$ may be charged, levitated and transported by the sheath of the parent object.

We have constructed an experiment to investigate photoelectric charging of isolated grains and those near surfaces. Experimental work on the photoelectric effect has been focused upon determination of the photoelectron energy distribution function through the use of a retarding potential and upon measurement of photoelectric yields [5]. For example, photoelectron yields as a function of wavelength have been determined for spacecraft materials [6]. The existence of a photoelectron sheath 
has been inferred from measurements made on spacecraft. Electron energy analyzers have observed low energy photoelectrons which originate from the spacecraft and are returned to the surface by the sheath potential [7]. These analyzers also see ambient plasma particles accelerated toward the spacecraft by the spacecraft charging potential [8].

The electron sheath was first analyzed theoretically for a thermionically emitting surface such as the cathode of a vacuum tube [9]. The emitted electrons in this case have a Maxwell-Boltzmann distribution with an energy of order $0.1 \mathrm{eV}$ determined by the cathode temperature. These may create a potential well near the surface of a few tenths of an $\mathrm{eV}$ which would be difficult to detect experimentally. For the photoelectron sheath, however, the energy distribution has a width of several $\mathrm{eV}$ and the electrons have a well-defined high energy cutoff determined by the difference between the work function of the material and the short-wavelength cutoff of the spectrum. The sheath potential profile is found by solving simultaneously Poisson's equation and the Vlasov equation. For thermionic emission, the potential approaches the potential at infinity asymptotically, however, for the photoelectron sheath this potential is reached in a finite distance as a consequence of the finite spread in the distribution function. Solutions for the photoelectron sheath have been given for several model distribution functions $[10,11,12]$.

\section{EXPERIMENTAL SETUP AND RESULTS}

The experiments are performed in a device (Fig. 1) used previously for measurements of dust charging in plasma $[13,14,15]$. This device consists of two aluminum cylinders $30 \mathrm{~cm}$ in diameter and $30 \mathrm{~cm}$ long placed end to end. Experiments are carried out in one of the two sections. The chamber is pumped by a diffusion pump to a base pressure of $4 \times 10^{-7}$ Torr.

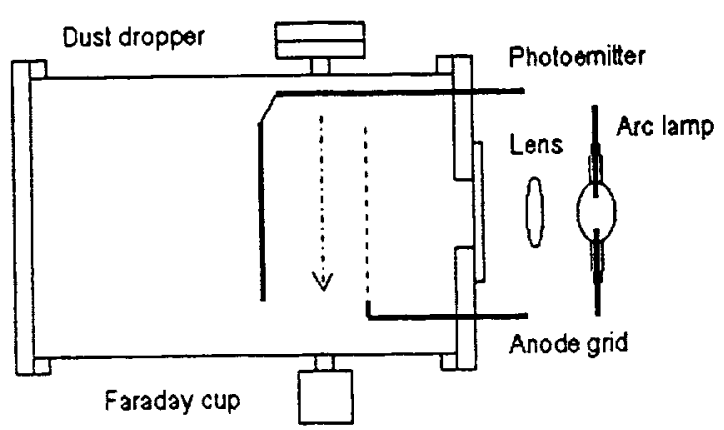

FIGURE 1. Schematic diagram of the experiment. The dust (dotted arrow) falls from a dropper at the top of the vacuum chamber then falls past a photoemitting surface. The charge on the dust is measured by a Faraday cup below the chamber. The photoemitter and the anode grid may be removed to determine the photoelectric charging of isolated grains. 
Photoelectric emission is induced by a $1 \mathrm{~kW} \mathrm{Hg-Xe} \mathrm{arc} \mathrm{lamp.} \mathrm{The} \mathrm{light} \mathrm{is}$ collimated by a lens and directed through a window into the vacuum system. The collection efficiency of the optics is such that about $10 \%$ of the lamp emission falls upon the photoemitter. All optical components are of quartz so that wavelengths down to $200 \mathrm{~nm}$ are passed. Approximately $1.4 \%$ of the lamp spectrum is in the wavelength band $200-250 \mathrm{~nm}$.

The photocathode is an electrically isolated $12.5 \mathrm{~cm}$ diameter zirconium foil disc. Zirconium and hafnium have the lowest work functions $(\sim 4 \mathrm{eV})$ of elements that are neither radioactive nor reactive in air [16]. Zirconium was found to give a slightly higher photoelectric yield with up to $20 \mu \mathrm{A}$ being obtained. For some experiments, an anode grid of nickel wires $15 \times 15 \mathrm{~cm}^{2}$ is placed parallel to the photoemitter.

The energy distribution of the photoelectrons is determined by a retarding potential analysis. All potential measurements are made relative to a copper grounding strap. The potential within the grounded aluminum chamber differs from this ground due to contact potentials. The potential in the vacuum adjacent to the grounded photoemissive surface, for example, is about one volt positive relative to the potentials adjacent to other surfaces because the photocathode has a lower work function. For the retarding potential analysis, the photocathode is swept in voltage and the emitted current is measured. The anode mesh is spaced $2.5 \mathrm{~cm}$ from the photocathode and is held at -4.5 volts so that any low energy electrons from beyond the mesh are accelerated toward the wall. The foil is illuminated in a central region $8 \mathrm{~cm}$ in diameter to minimize electron losses from the edge.

Figure 2a shows the photocathode current as a function of bias potential. For this measurement, the photoemission is reduced to $2 \mu \mathrm{A}$ to reduce space-charge effects which would alter the measurement. At a photocathode potential 1 volt more negative than the anode nearly all of the emitted electrons pass to the anode. At a potential 1 volt more positive, nearly all of the electrons are returned. This point would occur at a potential two volts more positive if there were no contact
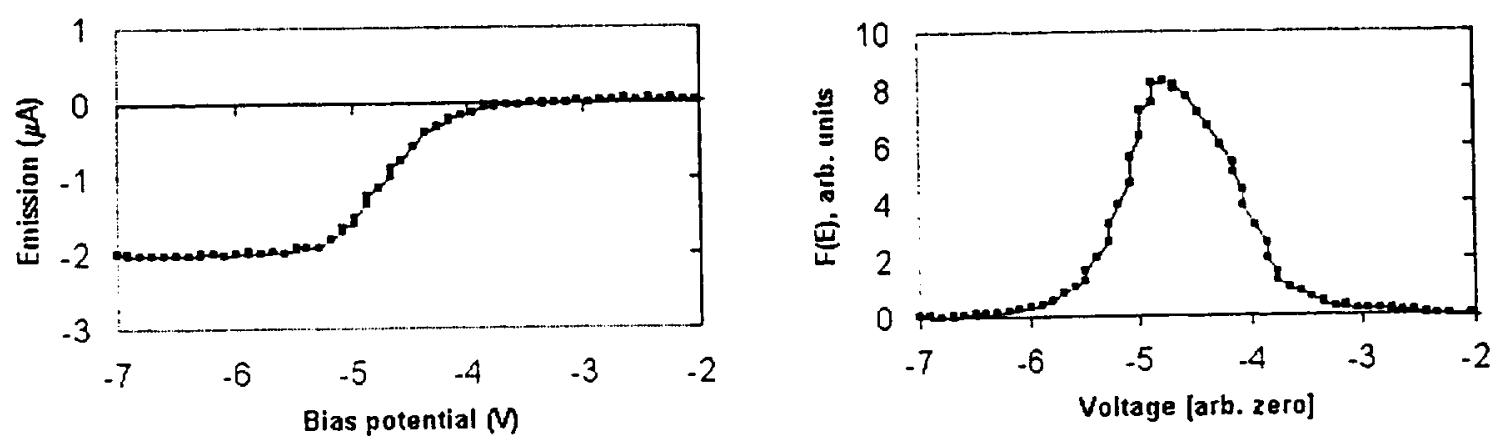

FIGURE 2. a) (left) Current emitted by the zirconium photoemitter (in microamps) as a function of its bias potential. The anode is at -4.5 volts. b) (right) The electron energy distribution obtained from the derivative of the curve in a). The low energy end of the distribution is on the left side. 
potential. Figure $2 \mathrm{~b}$ shows the derivative of the retarding potential curve which gives the distribution of electron energies perpendicular to the surface. The full width of the curve is approximately $2 \mathrm{eV}$ which indicates a mean electron energy of approximately $1 \mathrm{eV}$. A maximum electron energy of $2.15 \mathrm{eV}$ is expected from the difference between the short wavelength cutoff in the spectrum of illumination $(6.2 \mathrm{eV})$ and the work function of zirconium $(4.05 \mathrm{eV})$.

The density of photoelectrons above the surface can be estimated using the emitted electron current density and the mean electron velocity. If we assume an effective emission area of $10 \mathrm{~cm}$ in diameter the emission is $2.5 \times 10^{-3} \mathrm{~A} / \mathrm{m}^{2}$. If we further assume a mean electron velocity of $6 \times 10^{5} \mathrm{~m} / \mathrm{s}$ corresponding to an energy of $1 \mathrm{eV}$, the density of photoelectrons above the photoemitter is $2.6 \times 10^{4} \mathrm{~cm}^{-3}$. This value is doubled if the surface is biased relative to the surroundings so that electrons are returned.

The dust particles are of powdered zinc that is sieved to obtain particles with diameters of 53-63 microns. In the absence of the photoemitting surface, these particles become charged positively by their own photoemission. Experiments with a zinc surface in the place of the zirconium surface indicate that the emission from zinc is $10 \%$ of that from zirconium or $2.5 \times 10^{-4} \mathrm{~A} / \mathrm{m}^{2}$. The photoelectric work function of zinc $(4.3 \mathrm{eV})$ is near that of zirconium so we expect that the zinc will charge positively by two volts. The capacitance of the grains $\left(3.2 \times 10^{-15} \mathrm{~F}\right)$ is such that we expect a charge of $2.0 \times 10^{4}$ electrons per volt or approximately 4 $\times 10^{4}$ electrons. This value is near the threshold of detection. The time for the zinc grains to charge can be calculated from the expected emission current and the capacitance and is approximately 0.01 seconds, which is short in comparison with the time the dust is within the beam $(0.1 \mathrm{sec})$.

The particles are dropped through the illumination beam and captured in the Faraday cup. The signal from the Faraday cup has been calibrated to yield charge
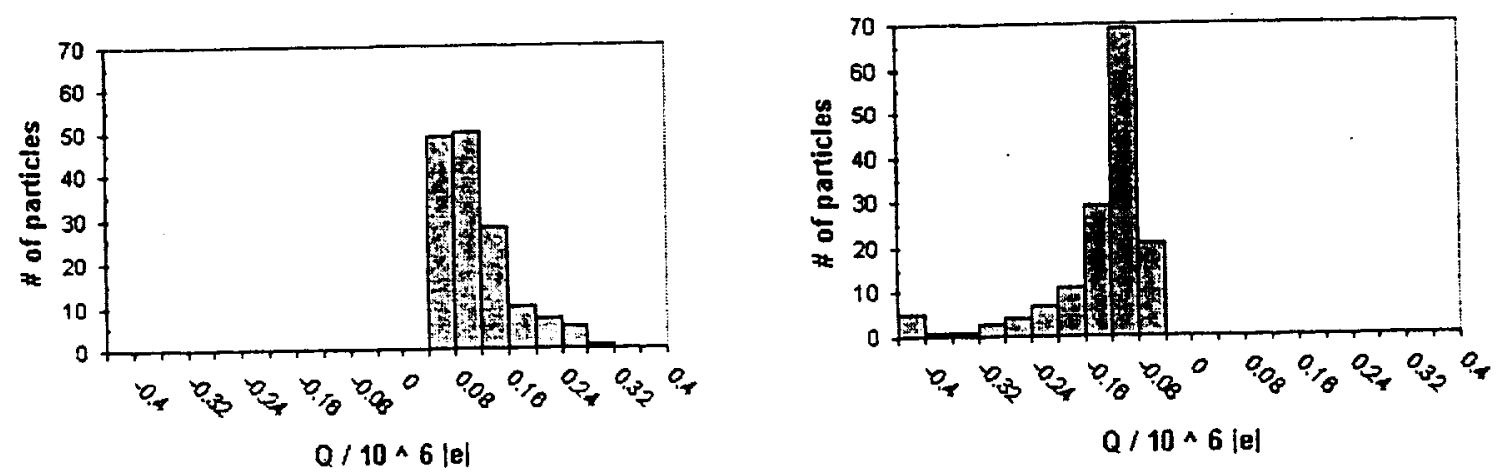

FIGURE 3. a) (left) Histogram of charge (in number of elementary charges) for 150 zinc dust particles dropped through the illumination beam with the photocathode removed. b) (right) Histogram of charge with the photocathode present and illuminated. Positive charge is a positive number for these graphs and the dust diameters are 53-63 microns. 
as a function of pulse height. The detected charge is sorted into bins with a width of $4 \times 10^{4}$ electrons (Fig. 3a). The two histogram bins adjacent to zero cannot be used because of false triggers from the circuit noise. In the absence of illumination, dropped dust results in no triggers. With illumination and no photoemitting plate, each of 150 dropped particles has a positive charge with the bins from $4 \times 10^{4}$ to $12 \times 10^{4}$ having the most particles. The larger charge on some particles is probably a result of the circuit noise adding to the true signal. A careful statistical analysis is required to remove this effect or a better detection system.

Data for grains dropped past the photoemitting plate are shown in Figure $3 \mathrm{~b}$. In this case each of 150 particles charges negatively with the greatest number of charges being between $8 \times 10^{4}$ and $12 \times 10^{4}$. The current density above the zirconium surface is about ten times larger than the current density emitted from the zinc surface, thus the charging is dominated by electron collection rather than by photoemission from the grains. Again the data are affected by circuit noise.

\section{FUTURE EXPERIMENTS}

We plan to continue these experiments using materials of space research interest. We will use simulated as well as real lunar Apollo 17 samples to understand the charging and dynamics of levitated dust clouds on the Moon. We also plan to conduct experiments using simulated Mars regolith. We are currently building a new experimental chamber where the photoemitting surface will be horizontal so that the sheath electric field can balance gravity on the charged dust particles. The properties of the sheath will be studied as function of the dust density and size distribution. This setup is the laboratory analog for future space station experiments to study dusty plasma sheaths in microgravity environments.

\section{ACKNOWLEDGMENTS}

The authors acknowledge support from the National Aeronautics and Space Administration and the Department of Energy.

\section{REFERENCES}

1. E. C. Whipple, Rep. Prog. Phys. 44, 1197 (1981).

2. S. F. Singer and E. H. Walker, Icarus 1, 112 (1962).

3. P. Lee, Icarus 124, 181 (1996).

4. T. Nitter and O. Havnes, Earth, Moon and Planets 56, 7 (1992).

5. A. L. Hughes and L. A. DuBridge, Photoelectric Phenomena, McGraw Hill, 1943.

6. B. Feuerbacher and B. Fitton, J. Appl. Phys. 43, 1563 (1972).

7. E. C. Whipple, Jr., J. Geophys. Res. 81, 815 (1976).

8. S. E. DeForest, J. Geophys. Res. 77, 651 (1972). 
9. The Collected Works of Irving Langmuir, C. G. Suits, ed., Pergamon, New York, 1961. Vols. 3 and 4.

10. S. F. Singer and E. H. Walker, Icarus 1, 7 (1962).

11. E. Walbridge, J. Geophys. Res. 78, 3668 (1973).

12. R. J. L. Grard and J. K. E. Tunaley, J. Geophys. Res. 76, 2498 (1971).

13. B. Walch, M. Horányi, and S. Robertson, IEEE Trans. Plasma Sci. 22, 97 (1994).

14. B. Walch, M. Horányi and S. Robertson, Phys. Rev. Lett. 75, 838 (1995).

15. M. Horányi, B. Walch, S. Robertson and D. Alexander, J. Geophys. Res. 103, 8575 (1998).

16. H. B. Michaelson, J. Appl. Phys. 48, 4729 (1977). 